\title{
Influence of temperature-dependent properties on a gravity-driven thin film along inclined plate
}

https://doi.org/10.1515/nleng-2020-0002

Received Feb 27, 2019; accepted Aug 27, 2019.

\begin{abstract}
A numerical investigation into the effect of temperature-dependent fluid properties on the thin film flow along an inclined heated plate is presented. The equations governing the coupled flow and heat transfer are formulated based on couple stress non-Newtonian model. Solutions of the coupled nonlinear differential equations are tackled numerically by using the combination of shooting method and the Fehlberg-Runge-Kutta method. Findings are presented graphically and discussed precisely.
\end{abstract}

Keywords: variable properties; thin film; heated plate; free and adiabatic conditions

\section{Introduction}

The importance of thin fluid flow along an inclined plate in many physical and engineering environment has accounted for the increasing number of studies in recent times. These studies are necessary to provide better understanding and improvement in the theory and applications of many real-life scenarios that are based on thin films. Some of the examples includes photography, heat exchangers, lubrications, electroplating of surfaces, and synovial movements. In view of this, Kay et al. [1] presented the thin film flow analysis over heated inclined plane using environmental model approach. Alharbi and Naire [2] introduced the R-adaptive mesh method for solv-

\footnotetext{
*Corresponding Author: Samuel O. Adesanya, Department of Mathematical Sciences, Redeemer's University, Ede, Nigeria Environmental Hydrodynamics Unit, African Centre of Excellence for Water and Environmental Research, Redeemer's University, Nigeria, E-mail: adesanyas@run.edu.ng

Amanze C. Egere, Department of Mathematical Sciences, College of Natural Sciences, Redeemer's University, Nigeria Joel C. Ukaegbu, Department of Mathematics, Faculty of Science, Adeleke University, Ede, Nigeria, E-mail: ukaegbu.joel@adelekeuniversity.edu.ng Ramoshweu S. Lebelo, Department of Education, Vaal University of Technology, Vanderbijlpark, South Africa, E-mail: sollyl@vut.ac.za
}

ing spreading thin fluid by taking the surface tension at the free surface into consideration. In a study by Shuaib et al. [3] a numerical study of a thin fluid flow with a leakage was presented along an inclined surface. Yu and Cheng [4] presented experimental findings of the thin fluid flow behavior on the inclined plate. Du et al. [5] performed an experiment on the gravity driven thin film of water under constantly changing inclination along an ellipsoidal heated plate. More interesting results can be found in ref. [6-10] and references cited within.

Motivated by several findings of many thermal and mechanical engineering applications, the present article is devoted to temperature dependent properties of thin film flow. Since findings [11-16] documented in the literature have shown over the last few years that formulations based on constant fluid properties are assumptions that are only valid in cases when the heat flow has been neglected or in small but finite thermal ranges. In the context of thin film flow, several works have been documented in the literature on thermal instability with or without temperaturedependent properties. Closely related to this work is the brilliant effort by Makinde [16, 17], in which thin film along heated plate with inclination and the thermal instabilities associated with the gravity-driven flow was accurately determined based on aspect ratio approximation of the governing equations. However, apart from the dynamic viscosity, other fluid properties are very sensitive to temperature changes as confirmed in [11-16]. As a result, the specific objective of this article is to investigate the effect of variation of thermal conductivity, fluid temperature and internal heat generation on the flow and thermal structure of the thin gravity-driven flow which to the best of our understanding has not been addressed in spite of its various application in the thermal and lubrication community. The present work is coupled and nonlinear, therefore, the exact solution of the problem is not possible. The numerical solution of the problem has been obtained by using the combination of shooting method to convert the boundary value problem to its equivalent initial value problem and solved using the fourth order Runge-Kutta integration scheme. The result revealed the non-existence of solution as the critical values of the nonlinear terms are exceeded. 
The next section of the article is devoted to the mathematical analysis, which consist of the model formulation, dimensionless process and the numerical solution of the couple problem including thermal stability. Numerical results are presented in the third section of the paper while the article is concluded in section four.

\section{Mathematical analysis}

Consider the gravity-driven flow of a temperaturedependent viscous and incompressible fluid down an inclined heated surface. The heated plate is assumed to be at the angle of inclination, $\vartheta$, to the horizontal with uniform film thickness $\delta$ as shown in Figure 1 below. The fluid dynamic viscosity, thermal conductivity, and the internal heat generation depends on temperature.

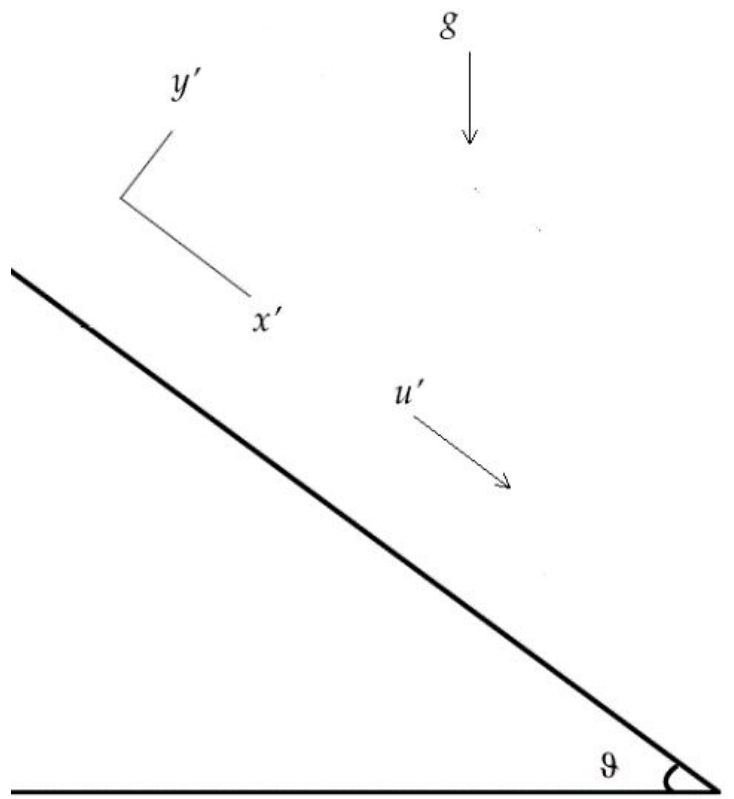

Figure 1: Flow geometry

Using the aspect ratio condition, the balance equations for the wave-less flow can be written as [16]:

$$
\begin{gathered}
0=\frac{d}{d \bar{y}}\left(\mu \frac{d u}{d \bar{y}}\right)+\rho g \sin \vartheta \\
0=\frac{d}{d \bar{y}}\left(k \frac{d T}{d \bar{y}}\right)+\frac{\mu}{k}\left(\frac{d u}{d \bar{y}}\right)^{2}+Q
\end{gathered}
$$

Subject to free and adiabatic flow conditions

$$
\begin{aligned}
& \bar{u}=0, \quad T=T_{0} \quad \text { on } \quad \bar{y}=0 \\
& \frac{d \bar{u}}{d \bar{y}}=0, \quad \frac{d T}{d \bar{y}}=0 \quad \text { on } \quad \bar{y}=\delta
\end{aligned}
$$

Additional terms in (1)-(3) are temperature dependent internal heat generation and thermal conductivity. In equations (1)-(3), $u, \rho, g$ represent the fluid dimensional velocity, density, and gravitational acceleration respectively, the fluid dynamic viscosity $\mu$, thermal conductivity $\mathrm{k}$ depends on temperature $\mathrm{T}$ in an exponential manner while internal heat generation $Q$ is a linear function as follows:

$$
\mu=\mu_{0} e^{-\alpha^{\prime}\left(T-T_{a}\right)}, k=k_{0} e^{-\beta^{\prime}\left(T-T_{a}\right)}, Q=Q_{0}\left(T-T_{0}\right)
$$

where $\mu_{0}, k_{0}, T_{a}$ are the referenced dynamic viscosity, thermal conductivity, and temperature (associated to a) respectively. Also, $\alpha^{\prime}, \beta^{\prime}, Q_{0}$ are variation constants which could take positive or negative values depending on the fluid under consideration. Using the following dimensionless variables and parameters

$$
\begin{gathered}
y=\frac{\bar{y}}{\delta}, \quad U=\frac{\mu_{0} u}{\delta^{2} \rho g \operatorname{Sin} \vartheta}, \theta=\frac{T-T_{a}}{T_{a}}, \alpha=\alpha^{\prime} T_{a}, \\
\beta=\beta^{\prime} T_{a}, \lambda=\frac{\left(\delta^{2} \rho g \operatorname{Sin} \vartheta\right)^{2}}{\mu_{0} T_{a} k}, \eta=\frac{\delta^{2} Q_{0}}{k_{0}}
\end{gathered}
$$

we get

$$
\begin{aligned}
\frac{d^{2} U}{d y^{2}} & =\alpha \frac{d \theta}{d y} \frac{d U}{d y}-e^{\alpha \theta} ; \quad U(0)=0=\frac{d U}{d y}(1) \\
\frac{d^{2} \theta}{d y^{2}} & =\beta\left(\frac{d \theta}{d y}\right)^{2}-\lambda e^{(\beta-\alpha) \theta}\left(\frac{d U}{d y}\right)^{2}-\eta \theta e^{\beta \theta} ; \\
\theta(0) & =0=\frac{d \theta(1)}{d y}
\end{aligned}
$$

Here the dimensionless parameters $U, \alpha, \theta$ are dimensionless velocity, viscosity variation, and dimensionless temperature respectively while $\beta, \lambda, \eta$ are thermal conductivity variation, viscous heating and internal heat generation parameter respectively. Moving forward, the boundary value problem (BVP) in (6)-(7) are coupled and nonlinear. The exact solution of the problem is practically not possible; therefore, it is more convenient to obtain the numerical solution. We implement the famous shooting method on the coupled system by converting the boundary value problem to its equivalent initial value problem as follows.

$$
x_{1}=U, x_{2}=U^{\prime}, x_{3}=\theta, x_{4}=\theta^{\prime}
$$

With (8), the coupled BVP becomes

$$
\left(\begin{array}{l}
x_{1} \\
x_{2} \\
x_{3} \\
x_{4}
\end{array}\right)^{\prime}=\left(\begin{array}{l}
x_{2} \\
\alpha x_{4} x_{2}-e^{\alpha x_{3}} \\
x_{4} \\
\beta x_{4}^{2}-\lambda e^{(\beta-\alpha) x_{3}}-\eta x_{3} e^{\beta x_{3}}
\end{array}\right)
$$


Where (') represents derivative with respect to $x_{1}$, equation (9) is subject to the initial conditions

$$
\left(\begin{array}{l}
x_{1}(0) \\
x_{2}(0) \\
x_{3}(0) \\
x_{4}(0)
\end{array}\right)=\left(\begin{array}{l}
0 \\
D_{1} \\
0 \\
D_{2}
\end{array}\right)
$$

The system of equations (9) together with the initial conditions are elegantly obtained by using Runge-Kutta with the Fehlberg numerical integration approach. It is important to note that $D_{1,2}$ are the guess values that ensures that the boundary conditions at $y=1$ are met.

Next, the skin interaction and the wall heat transfer rate along the heated wall can be expressed as follows:

$$
\tau_{w}=\left.\mu_{0} e^{-\alpha^{\prime}\left(T-T_{a}\right)} \frac{d u}{d y^{\prime}}\right|_{y^{\prime}=0}
$$

With (5), (11) becomes

$$
S f=\frac{\tau_{w}}{\delta \rho g \sin \alpha}=\left.e^{-\alpha \theta} \frac{d U}{d y}\right|_{y=0}
$$

\section{Results and discussion}

In this section, graphical representations of the numerical solutions are presented for various flow parameter values. In Figure 2, the temperature profile is represented against the thermal conductivity varying parameter. From the plot, rise in the variable thermal conductivity parameter leads to increase in the rate of heat transfer at the free end due to increasing heat flux, however, the heat at inclined plate affects the thermal conductivity of the fluid layer closer to the wall and that explains the minimal convective heat transfer in that region. Results in Figure 3 shows that convective heat transfer rate elevates the fluid velocity, and this follows the same trend with the temperature profile. In Figure 4, the effect of viscosity varying parameter on the temperature profile is presented. Result shows that decreasing fluid viscosity enhances the fluid temperature. This is correct because of increased molecular interaction of the fluid particles associated with decrease in the cohesive forces as the fluid viscosity decreases. Similarly, in Figure 5, thermal effect on the fluid viscosity is presented. From the result, increased viscosity variation parameter weakens cohesive force binding the fluid particles, as a result, the increased kinetic energy of the fluid particles enhances the deformation rate as shown in the plot.

Figures 6 and 7 represent the effect of viscous heating on the fluid temperature and velocity respectively. The

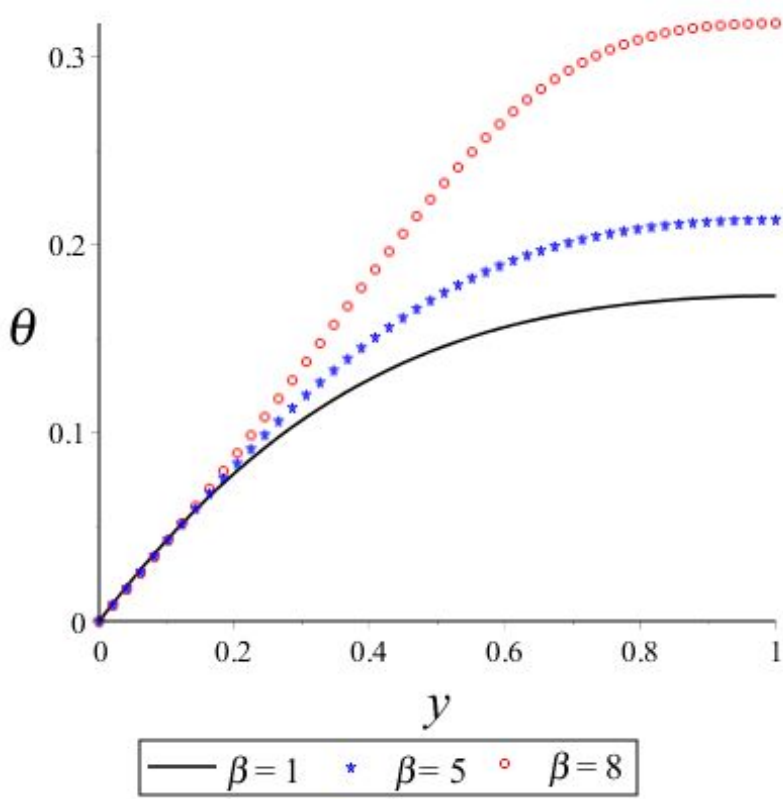

Figure 2: $\beta$ effect on temperature

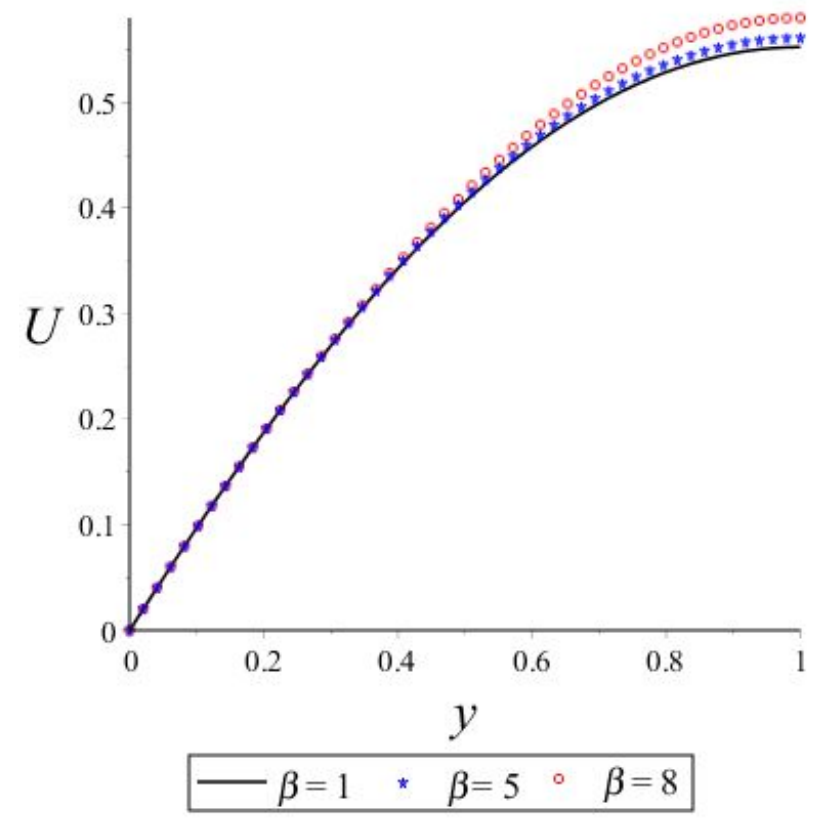

Figure 3: $\beta$ effect on velocity

result shows heat generated from frictional interaction of fluid particles elevates the fluid temperature and the velocity respectively. Figures 8 and 9 depict the influence of temperature dependent internal heat generation parameter on the temperature and velocity profile respectively. From the plots, increasing the internal heat generation parameter helps elevate both fluid temperature and the thin film velocity along the heated plate. In Figure 10, the varia- 


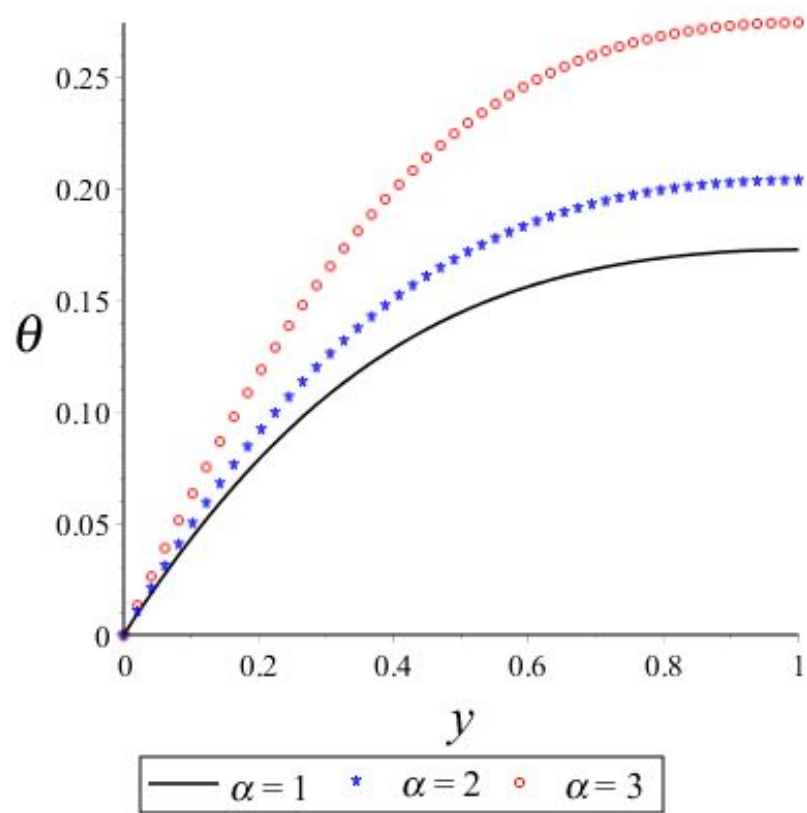

Figure 4: $\alpha$ effect on temperature

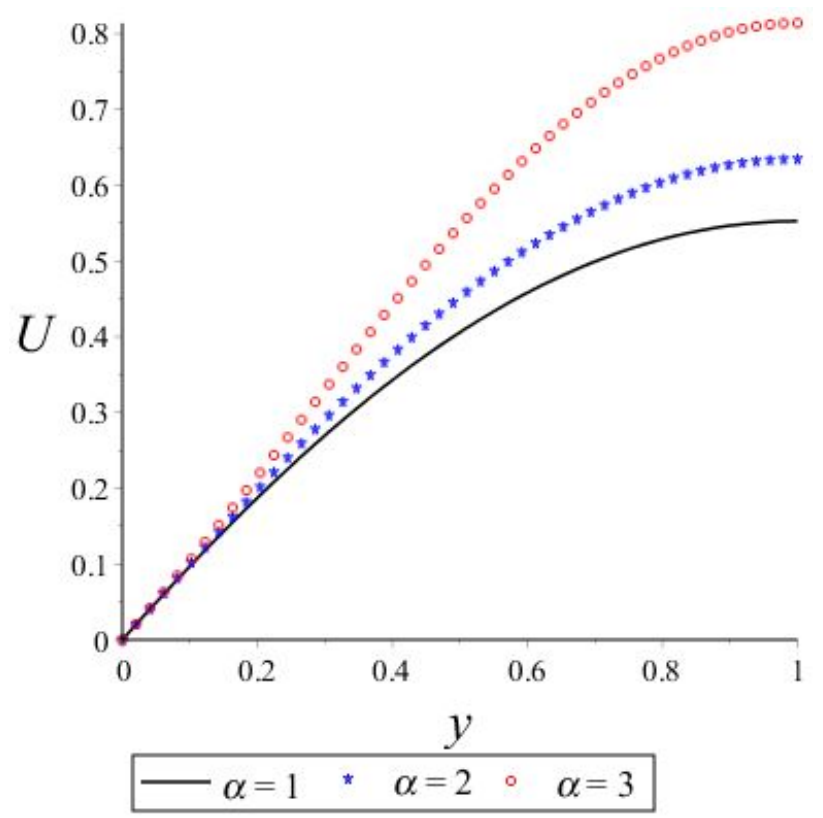

Figure 5: $\alpha$ effect on velocity

tion of wall heat transfer $\left(\theta_{\max }\right)$ with $\beta$ and $\alpha$ is illustrated. An increase in $\beta$ values shows a decrease in the criticality values $\left(\alpha_{c}\right)$, and this means that the rate of heat transfer at the wall is reduced as the viscosity of the fluid is increased, the same scenario is observed in Figure 11.

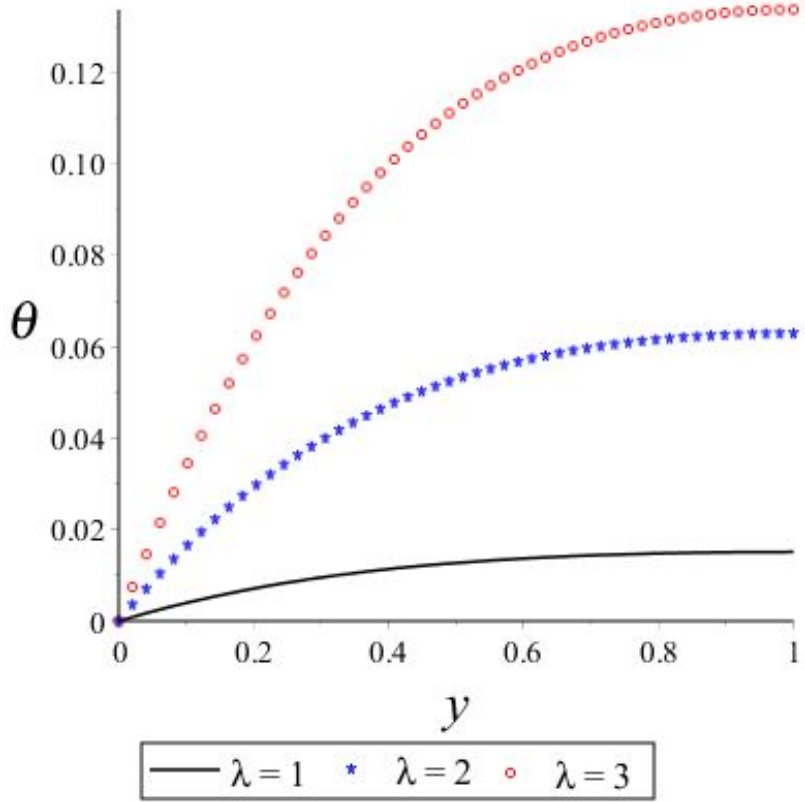

Figure 6: $\lambda$ effect on temperature

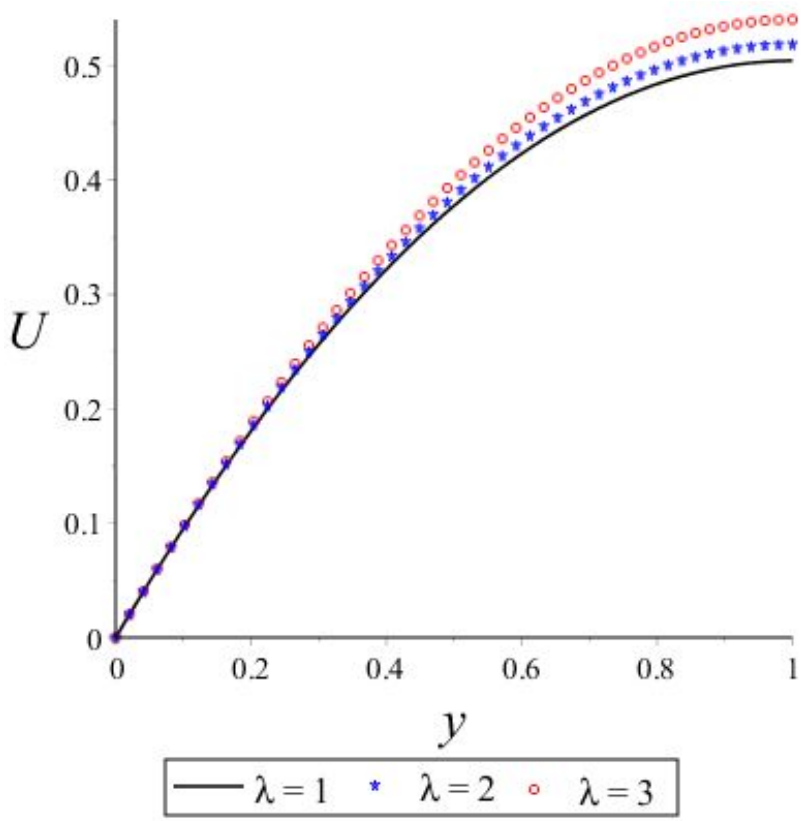

Figure 7: $\lambda$ effect on velocity

\section{Conclusion}

A numerical approach to investigate the thin film flow with temperature-dependent properties along heated inclined plates subjected to free and adiabatic surface has been presented. From the numerical computation, the temperature-dependent heat source, viscosity and thermal conductivity parameters are seen to enhance both 


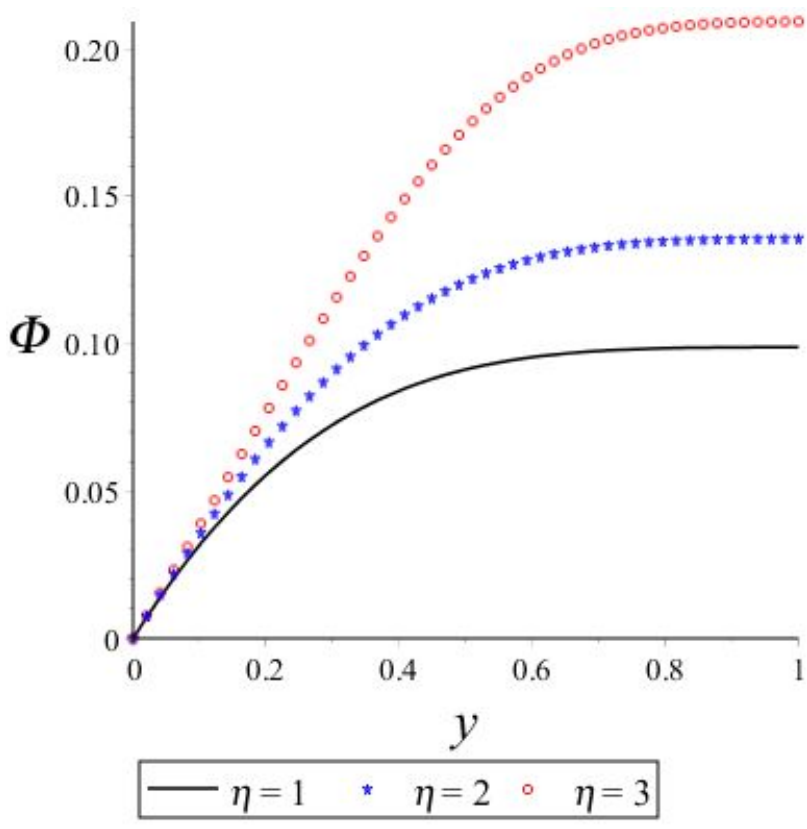

Figure 8: $\eta$ effect on temperature

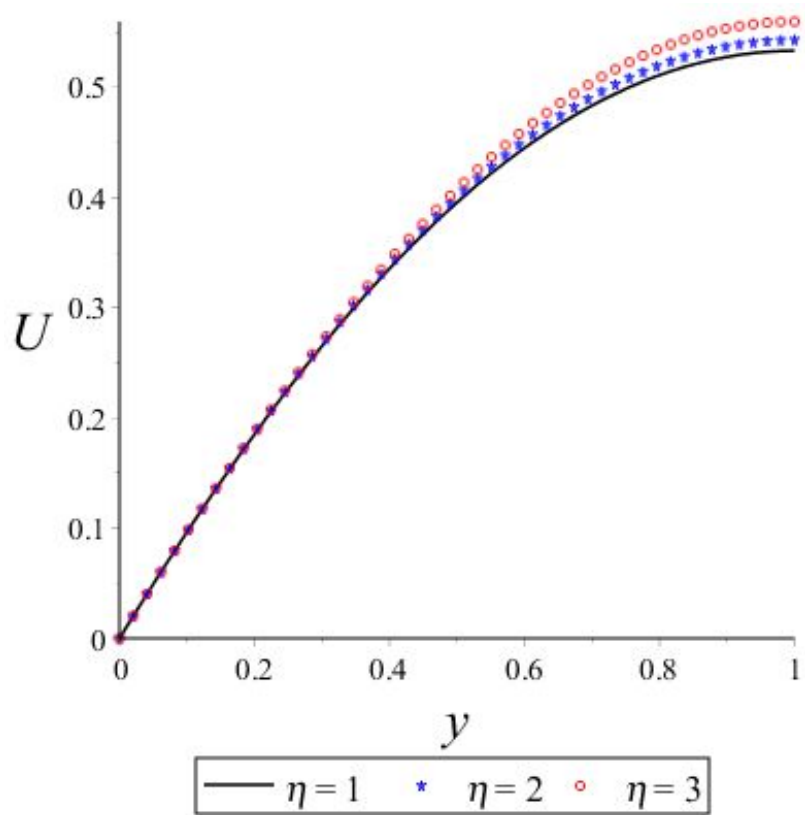

Figure 9: $\eta$ effect on velocity

fluid velocity and temperature significantly. Area of future research is not limited to the computation of critical values for thermal runaway.

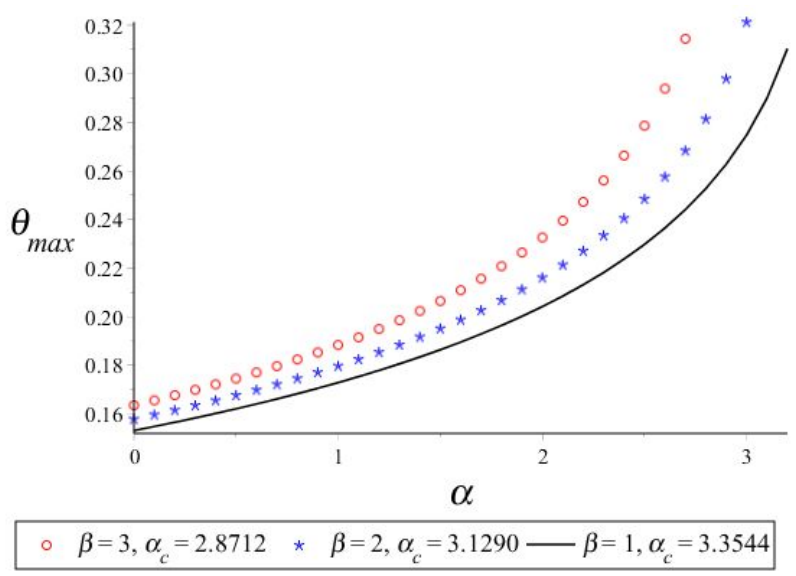

Figure 10: Wall heat transfer rate variation with $\beta$ and $\alpha$

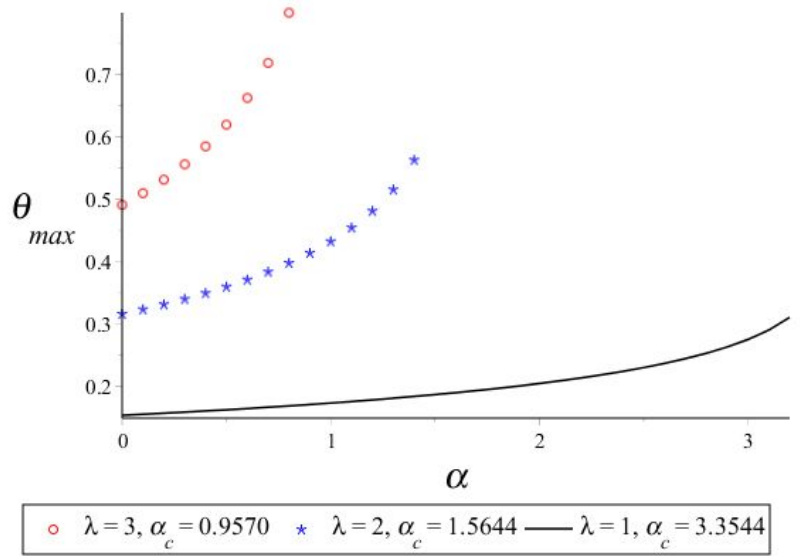

Figure 11: Wall heat transfer rate variation with $\lambda$ and $\alpha$

\section{References}

[1] Kay E.D., Hibberd S., Power H., A multi-layer integral model for locally-heated thin film flow, J. Comput. Phys., 2017, 336, 51-68.

[2] Alharbi A., Naire S., An adaptive moving mesh method for thin film flow equations with surface tension, J. Comput. Appl. Math., 2017, 319, 365-384.

[3] Shuaib N.H., Power H., Hibberd S., BEM solution of thin film flows on an inclined plane with a bottom outlet, Eng. An. Bound. Elem., 2009, 33, 388-398.

[4] Yu Y.Q., Cheng X., Experimental study of water film flow on large vertical and inclined flat plate, Progr. Nucl. Energy, 2014, 77, 176-186.

[5] Du W.-F., Lu Y.-H., Zhao R.-C., Chang L., Chang H.-J., Film thickness of free falling water flow on a large-scale ellipsoidal surface, Progr. Nucl. Energy, 2018, 105, 1-7.

[6] Chinak A.V., Gorelikova A.E., Kashinsky O.N., Pakhomov M.A., Randin V.V., Terekhov V.I., Hydrodynamics and heat transfer in an inclined bubbly flow, Int. J. Heat Mass Transf., 2018, 118, 785-801. 
[7] Sivapuratharasu M., Hibberd S., Hubbard M.E., Power H., Inertial effects on thin-film wave structures with imposed surface shear on an inclined plane, Physica D, 2016, 325, 86-97.

[8] Shri Vignesh K., Vasudevan C., Arunkumar S., Suwathy R., Venkatesan M., Laser induced fluorescence measurement of liquid film thickness and variation in Taylor flow, Europ. J. Mech. / B Fluids, 2018, 70, 85-92.

[9] Kofman N., Rohlfs W., Gallaire F., Scheid B., Ruyer-Quil C., Prediction of two-dimensional dripping onset of a liquid film under an inclined plane, Int. J. Multiphase Flow, 2018, 104, 286-293.

[10] Zhang H., Zegeling P.A., Simulation of thin film flows with a moving mesh mixed finite element method, Appl. Math. Comput., 2018, 338, 274-289.

[11] Anjali Devi S.P., Prakash M., Temperature dependent viscosity and thermal conductivity effects on hydromagnetic flow over a slendering stretching sheet, J. Nigerian Math. Soc., 2015, 34, 318-330.

[12] Abd El-Aziz M., Temperature dependent viscosity and thermal conductivity effects on combined heat and mass transfer in MHD three-dimensional flow over a stretching surface with Ohmic heating, Meccanica, 2007, 42, 375-386.

[13] Sharma P.R., Singh G., Effects of varying viscosity and thermal conductivity on steady MHD free convective flow and heat transfer along an isothermal plate with internal heat generation, Int. J. Numer. Meth. Heat \& Fluid Flow, 2009, 19(1), 78-92.

[14] Keimanesh R., Aghanajafi C., the effect of temperaturedependent viscosity and thermal conductivity on micropolar fluid over a stretching sheet, Tehnički vjesnik 2017, 24(2), 371-378.

[15] Parveen N., Alim M.A., Numerical solution of temperature dependent thermal conductivity on mhd free convection flow with joule heating along a vertical wavy surface, J. Mech. Eng., 2014, 44(1), 43-50.

[16] Elbashbeshy E.M.A., Bazid M.A.A., The effect of temperaturedependent viscosity on heat transfer over a continuous moving surface, J. Phys. D: Appl. Phys., 2000, 33, 2716-2721.

[17] Makinde O.D., Hermite-Pade approximation approach to steady flow of a liquid film with adiabatic free surface along an inclined heat plate, Physica A, 2007, 381, 1-7.

[18] Makinde O.D., Laminar falling liquid film with variable viscosity along an inclined heated plate Applied Mathematics and Computation, 2006, 175, 80-88.

[19] Makinde O.D., Thermal criticality for a reactive gravity driven thin film flow of a third grade fluid with adiabatic free surface down an inclined plane, Appl. Math. Mech. Engl. Ed., 2009, 30(3), 373-380. 